\title{
Effects of rehabilitation therapy in patients with psoriatic arthritis
}

\author{
Diana Maghiar ${ }^{1}$, Nicoleta Anamaria Pascalau ${ }^{2,3}$, Florin Marcu, ${ }^{2,4,}$ Liviu Lazar $^{1,2,4}$ \\ ${ }^{1}$ Doctoral Studies Department, Biomedical science, Oradea, Romania \\ ${ }^{2}$ Department of Medicine-Psycho-Neuroscience and Recovery, Faculty of Medicine and Pharmacy, \\ Oradea, Romania \\ ${ }^{3}$ Pelican Hospital, Oradea, Romania \\ ${ }^{4}$ Baile Felix Medical Rehabilitation Hospital, Baile Felix Resort, Bihor County, Romania
}

\begin{abstract}
Objective. Our main goal in this study was to determine whether or not in addition to classical and biological treatments, rehabilitation treatment had any benefit in the evolution of the psoriatic arthritis.

Material and methods. We evaluated two groups of patients, classified according to their basic therapies, namely classical treatment of psoriatic arthritis (methotrexate) and biological treatment respectively. Besides drug treatment, one of the groups (study group) received rehabilitation treatment with different methods of application (hydrotherapy, kinetotherapy).

Outcomes. An important result driven from the data evaluation was that patients who, in addition to the background treatment, whether biological treatment or classic, had also associated the rehabilitation therapy had better results in improving the quality of life and reducing the progression of the inflammatory syndrome (DLQI had better results in $93.1 \%$ in the study group vs $68.97 \%$ in control group).

Conclusions. Seeing only the benefits of rehabilitation therapy, we can say that, if it were added to the patient's treatment plan, it would lead to a slow evolution of the disease and a change in the quality of life for the better.
\end{abstract}

Keywords: psoriatic arthritis, biological treatment, rehabilitation, clinical evaluation

\begin{abstract}
Abbreviations
PsA - psoriatic arthritis

DAPSA - disease activity in psoriatic arthritis

CASPAR - the classification criteria for psoriatic arthritis

DLQI - dermatology life quality index

HRQoL - health-related quality of life

PASI - psoriasis area severity index

ESR - erythrocyte sedimentation rate

$\mathrm{CRP}$ - C-reactive protein
\end{abstract}

\section{INTRODUCTION}

Psoriatic arthritis (PsA) is a chronic disease which involves the inflammation of synovial tissue, entheses, skin and is usually seronegative for rheumatoid factor. PsA patients have heterogeneous clinical presentations, with diverse articular and dermatological features and varied disease courses and outcomes. PsA was initially considered to be a mild disease, but in the past decade $40 \%-60 \%$ of patients have developed erosive and deforming joint complications (1).
Over the past 15 years the therapeutic options for severe and generalized psoriasis have broadened immensely with the introduction of biological agents to everyday practice. Due to distinctive pathological mechanisms leading to the formation of psoriatic lesions, in which the crucial role is played by the cell-mediated immune response, the advent of medications specifically modulating the adaptive immune response has become a milestone in treatment of moderate and severe psoriasis. Biological agents include fusion proteins and monoclonal antibodies, 
which are designed to target specific components of the immune system, usually by binding the mediators of inflammation or their respective receptors. High selectivity of targeted immunotherapy minimalizes the risk of adverse effects and enhances the effectiveness of treatment $(2,3)$.

Depending on the location and severity, psoriasis can be treated with topical agents (e.g. D-vitamin analogues, corticosteroids, dithranol), systemic medications (e.g. methotrexate, biological agents) and natural treatments (e.g. UV-light, peloid, medicinal water). The complex spa therapy used during the rehabilitation is an effective tool to reduce the symptoms of psoriasis and improve the patient's well-being.

The rehabilitation treatment program for patients with psoriatic arthritis should be individualized and should be started early in the disease process.

\section{AIM AND OBJECTIVES}

Our main goal in this study was to determine whether or not, in addition to classical and biological treatments, rehabilitation treatment has advantages in the evolution of the disease we studied. Thus, we identified the cases diagnosed with psoriatic arthritis and we observed their evolution before and after the rehabilitation treatment (DAPSA, DLQI and PASI score).

\section{MATERIAL AND METHODS}

In both groups, patients with co-morbidities that had contraindications to the thermal therapy, pregnant or nursing women, persons at risk of not being present at the prescribed controls (psychiatric disorders, deprivation of liberty) were not included in both groups.

Standard evaluation: corresponds to the collection of general patient data on name and surname, date of birth, address, contact data and socio-demographic data including age, sex, height, weight, current reproductive status, habitat, training level, professional activity, exposure to noxious factors (active and passive smoking, other toxic substances).

Patients of study group received thermal treatment (hydrotherapy), kinetotherapy, therapeutic massage or even paraffin wraps for 14 days at the Clinical Hospital of Rehabilitation Baile Felix. All patients were evaluated initial, at the admission to study, and after 6 months.

Standard evaluation used were: PASI score - psoriasis area and severity index; DAPSA score - dis- ease activity in psoriatic arthritis; DLQI - dermatology life quality index.

The statistical analysis was performed using SPSS 20. All the average parameter values, standard deviations, frequency ranges, and statistical significance tests were calculated by using the Student method - t-test and $\mathrm{c}^{2}$. ES was used to measure the sensitivity to change (ES $<0.2$ - without modification, $\mathrm{ES}=0.2-0.5$ - small modification, $\mathrm{ES}=0.5-0.8$ - moderate modification, ES $>0.8$ - great modification). A p $<0.05$ value was attributed to statistical significance.

We performed a comparative study regarding the evolution of 58 patients diagnosed with psoriatic arthritis with background therapy (classical or biological treatment) associated or not with rehabilitation program. Half of them followed balneo-physio-therapy associated with the background treatment, named study group, and the other half remains only with the background treatment, named control group.

Inclusion criteria for the study group

- patients who have had a thermal cure lasting at least 10 days at the beginning of the follow-up period of one year

- diagnosis of psoriatic arthritis defined by the specialist rheumatologist and dermatologist (according to CASPAR criteria);

- DAPSA score at inclusion of over 14 (moderate joint disease with DAPSA between 14 and 28 and high activity with DAPSA over 28)

- DLQI score at inclusion $\geq 10$

- the age of patients for at least 18 years

- onset/duration of symptoms for at least one year

- stable background treatment over the last 6 months

- patient consent to participate in the study

Inclusion criteria for the control group

- patients who did not receive thermal cure during the follow-up period of one year

- diagnosis of psoriatic arthritis defined by the specialist rheumatologist and dermatologist (according to CASPAR criteria);

- DAPSA score at inclusion over 14 (moderate activity with DAPSA between 14-28 and high activity with DAPSA over 28 of joint disease)

- DLQI score at inclusion $\geq 10$

- the age of patients for at least 18 years

- onset of symptoms for at least one year

- stable background treatment over the last 6 months

- patient consent to participate in the study 


\section{RESULTS}

In both groups most of patients were women $(58.62 \%$ vs $62.07 \%, \mathrm{p}=0.790)$ and the average age was significantly higher in study group (62.17 vs. 54.45 years, $\mathrm{p}<0.001$ ). About one third of patients had the duration of evolution of PsA over 5 years $(27.59 \%$ vs. $31.03 \%, \mathrm{p}=0.775)$.

It is well-known that psoriasis affects female sex more frequently. So its complications, like psoriatic arthritis, occur more often in women $(4,5)$.

Maybe the influence of treatment, or perhaps the role of the immune system still uninterrupted by the disease, have made the appearance of arthritis signs appear after a long period of time. On the other hand, there are patients with arthritis that undercome in less than 5 years from the diagnosis of psoriasis, in which case we can suspect an accelerated pathogenesis of the disease or the exaggerated involvement of some biomolecules (IL, inflammatory markers) (6).

Depending on the joint affection, it will be noticed that the most commonly affected joint is the distal inter-phalangeal joint of the hands (Fig. 1), followed by the involvement of the sacroiliac joint $(51.72 \%$ vs. $44.83 \%, p=0.603$, respectively $48.28 \%$ vs. $41.38 \%, \mathrm{p}=0.601)$.

Of course, a patient may have one or more sites of involvement. Although the most affected is the distal inter-phalangeal joint of the hand, affected sites that can also be seen are the scapula-humeral joint, elbow and knee.

In other studies sacroiliitis is a common finding among PsA patients: in a study made by Battistone et al. on the population of Italy showed that the prevalence of sacroiliitis was 32\% (1), and a multicenter study from the United States the prevalence of sacroiliitis was $78 \%$ (6). PsA can simulate rheumatoid arthritis to involve the knee or a large joint with some small joints in fingers or toes (7).

The evolution of patients of both groups has been analyzed in terms of severity of psoriasis, psoriatic arthritis activity index and quality of life. The severity of psoriasis was evaluated with PASI score.

At baseline, in almost $60 \%$ of patients in both groups the PASI score report moderate disease activity $(58.62 \%)$, and in the severe category we have $6.90 \%$ of patients. At baseline, the mean PASI score was 7.10 in study group, insignificantly higher than in control group $(6.90, \mathrm{p}=0.792)$.

At 6 months, the mean of PASI score decreased significantly in both groups (from 7.10 to 5.76, $\mathrm{p}=0.065$, respectively from 6.90 to $5.93, \mathrm{p}=0.217$ ), and the sensitivity to change, evaluated by effect size, reveals a small change in both groups, but higher in the study group $(\mathrm{ES}=0.45$, respectively $\mathrm{ES}=$ $0.35)$.

The PASI score was reduced in $79.31 \%$ in patients from the study group and in $58.62 \%$ in the control group ( $p=0.091)$, but the score increased in control group at $10.34 \%$ of patients.

At baseline, the activity of PsA was moderate in $93.10 \%$ patients from study group and in all patients from control group. The baseline DAPSA score was 18.72 in study group, insignificantly lower than in control group $(18.76, \mathrm{p}=0.960)$.

At 6 months, the mean of DAPSA score decreased significantly in both groups (from 18.72 to $16.62, \mathrm{p}=0.025$, respectively from 18.76 to 17.03 , $\mathrm{p}=0.046$ ), and the sensitivity to change, evaluated by effect size, reveals a moderate change in both groups, but higher in Study group ( $\mathrm{ES}=0.69$, respectively $\mathrm{ES}=0.56$ ).

The DAPSA score was reduced in 79.31\% from patients in study group and in $65.52 \%$ in control group $(\mathrm{p}=0.244)$, but the score increased in control group at $10.34 \%$ of patients.

Dermatology life quality index (DLQI) is the most commonly applied measure of health-related quality of life (HRQoL) in psoriasis patients. It is among defining criteria of moderate-to-severe psoriasis and present in treatment guidelines $(8,9)$.

At baseline, impact of PsA was very large and had extremely large effect (DLQI score $>10$ ) in $68.97 \%$ patients from study group and in $82.76 \%$ patients from control group $(\mathrm{p}=0.224)$. The baseline DLQI score was 12.14 in study group, insignificantly lower than in control group (13.55, $\mathrm{p}=0.108)$.

At 6 months, the mean of DLQI score decreased significantly in both groups (from 12.14 to 8.59 , p $<$ 0.001 , respectively from 13.55 to $11.21, \mathrm{p}=0.024$ ), and the sensitivity to change, evaluated by effect size, reveals a large change in study group and moderate in control group $(\mathrm{ES}=1.32$, respectively $\mathrm{ES}=$ $0.62)$.

The DLQI score was reduced in $93.10 \%$ from patients in study group and in $68.97 \%$ in control group $(\mathrm{p}=0.020)$, but the score increased in control group at $3.45 \%$ of patients.

To evaluate the inflammatory syndrome it was necessary to dose the C-reactive protein (CRP) and the erythrocyte sedimentation rate (ESR). After six months, at the check-ups, improvements have been 
seen in patients belonging to the Study group in $68.96 \%(E S=1.32)$. In the control group we noticed changes in the CRP value in the sense of decreasing its value in $58.62 \%$ of cases ( $68.96 \%$ study group vs. $58.62 \%$ control group). In the study group, $72.41 \%$ had lower ESR values compared to baseline values before the thermal cure started. In the control group $65.51 \%$ had decreased values of ESR (72.41\% study group vs $65.51 \%$ control group).

Physical therapy can play an important role in improving the life of a person with psoriatic arthritis. Physical therapy management should focus on education, improvement of range of motion, strengthening, and general cardiovascular conditioning. Physical therapists may also provide UV therapy and modalities to decrease pain. Cryotherapy may help to reduce swelling and tenderness in affected joints. Heat may be used to relieve joint pain. Paraffin baths tend to be soothing for the hands and feet. Splinting may be of benefit to prevent deformity.

Recently studies have shown that hydrotherapy is also an effective treatment for patients with psoriatic arthritis. Hydrotherapy has been shown to improve physical function, energy, sleep and relaxation, cognitive function, work, and participation in patients with psoriatic arthritis (10-13).

\section{DISCUSSIONS}

In the study performed by Ivan Peter et al. (2017), the results on patients with psoriasis and PsA are showing improvements in the CRP levels and PASI score after 3-week-long rehabilitation. The same results are shown in our study, with levels of CRP, ESR, PASI and even DAPSA score improved after the patients benefited from rehabilitation therapy (11).

Since there are not so many studies conducted to show the benefits of both rehabilitation and thermal therapy, we should consider also the fact that, besides the drug treatment used for this disease, patients could benefit from a kind of a non-drug treatment, that not improves only the inflammatory response but also their quality of life.

\section{REFERENCES}

1. Battistone MJ, Manaster BJ, Reda DJ, Clegg DO. The prevalence of sacroilitis in psoriatic arthritis: new perspectives from a large, multicenter cohort. A Department of Veterans Affairs Cooperative Study. Skeletal Radiol. 1999;28:196-201.

2. Mclnnes IB, Mease PJ, Kirkham B, Kavanaugh A, Ritchlin CT, Rahman P, et al. Secukinumab, a human anti-interleukin-17A

\section{Relevance to clinical practice}

Psoriatic arthritis is a slow but aggressive disease that affects patients previously diagnosed with psoriasis. The stigma that patients already have a dermatological disease to which is added arthropathy is a severe one leading to variable changes in the quality of life. Our study reveals that, in addition to the classic and biological treatment, the rehabilitation treatment has some advantages over the evolution of the disease as well as the psychosocial status. We believe that if this type of treatment is added, the lives of patients with psoriatic arthritis change into variables that go towards a good evolution of mental status and daily life.

\section{CONCLUSIONS}

Thermal therapy is used worldwide in the treatment of psoriasis but few controlled studies have evaluated its efficacy and safety. We decided to see its benefits on patients with psoriatic arthritis. From the two groups studied, the patients belonging to the group benefiting from balneotherapy (study group) had significantly improved results compared to those who remained strictly with background therapy (be it classic or biological treatment).

This study demonstrated the better results of rehabilitation therapy in patients with PsA, at 6 months, regarding the severity of psoriasis, the psoriatic arthritis activity score and the quality of life (both of the scores improved after rehabilitation therapy in more than $75 \%$ of patients).

In order to obtain more accurate results, being able to analyze more parameters, the study should be extended over o longer period, and the number of patients must be increased. Thermal cure as proved here, has a lot of positive benefits for patients suffering from this devastating disease, not only by showing clinical improvements, but also by having a significant impact on mental and quality of life of the patient.

\section{Acknowledgement}

All authors have equal implication in this study.

Conflict of interest: none declared Financial support: none declared

monoclonal antibody, in patients with psoriatic arthritis (FUTURE 2): A randomised, double-blind, placebo-controlled, phase 3 trial. Lancet. 2015;386 (9999):1137-46.

3. Nestle FO, Kaplan DH, Barker J. Psoriasis. N Engl J Med. 2009; 361(5):496-509. 
4. Brooks M. Ustekinumab approved for psoriatic arthritis in US, Europe. Available at https://www.medscape.com/ viewarticle/811496\#: :text=Ustekinumab\%20is\%20already $\% 20$ approved\%20in,\%2D12\%20and\%20IL\%2D23.

5. Duarte GV, Faillace C, Freire de Carvalho J, Psoriatic arthritis, Best Pract Res Clin Rheumatol. 2012;26(1):147-56.

6. Alinaghi F, Calov M, Kristensen LE, et al. Prevalence of psoriatic arthritis in patients with psoriasis: A systematic review and metaanalysis of observational and clinical studies. J Am Acad Dermatol. 2019;80(1):251-265.e19.

7. Payet J, Gossec L, Paternotte S, Burki V, Durnez A, Elhai M et al. Prevalence and clinical characteristics of dactylitis in spondylarthritis: A descriptive analysis of 275 patients. Clin Exp Rheumatol. 2012;30(2):191-6.

8. Punzi L, Pianon M, Rossini P, Schiavon F, Gambari PF. Clinical and laboratory manifestations of elderly onset psoriatic arthritis:
A comparison with younger onset disease. Ann Rheum Dis. 1999;58:226-229.

9. Dhir V, Aggarwal A. Psoriatic arthritis: A critical review. Clin Rev Allergy Immunol. 2013;44:141-148.

10. Cuchacovich R, Perez-Alamino R, Garcia-Valladares I, Espinoza LR. Steps in the management of psoriatic arthritis: A guide for clinicians. Ther Adv Chronic Dis. 2012;3(6):259-69.

11. Péter I, Jagicza A, Ajtay Z, et al. Balneotherapy in Psoriasis Rehabilitation. In Vivo. 2017;31(6):1163-1168.

12. Kelly JC. PsA Remission, Response Cutoffs Identified for DAPSA Tool. Available at https://www.medscape.com/viewarticle/850333.

13. Van den Bosch F, Coates $L$. Clinical management of psoriatic arthritis. Lancet. 2018;391(10136):2285-2294. 